\title{
Generalized Propagation Channel Model for 2GHz Low Elevation Links Using a Ray-tracing Method
}

\author{
Jan ZELENY ${ }^{1}$, Fernando PEREZ-FONTAN ${ }^{2}$, Pavel PECHAC \\ ${ }^{1}$ Dept. of Electromagnetic Field, Czech Technical University in Prague, Technická 2, 16000 Prague, Czech Republic \\ ${ }^{2}$ Dept. of Telecommunication, University of Vigo, Rua Maxwell, 36310 Vigo, Spain
}

\{zelenja3,pechac\}@fel.cvut.cz, fpfontan@tsc.uvigo.es

\begin{abstract}
Unmanned Aerial Vehicles (UAV) will increasingly be used for responding to emergencies or for law enforcement in civil surveillance applications. Transferring the enormous amounts of information from UAV-mounted cameras or sensors will require large bandwidths, unlike the information required for remotely controlling a UAV, thus necessitating higher frequency bands typically in the vicinity of 2 or $5 \mathrm{GHz}$. Novel hardware developments will need to rely on a versatile propagation channel model for the envisaged scenarios ranging from deep shadow urban areas to open fields. This paper studies more complex intermediate scenarios, which fall between the aforementioned ones, and which are more difficult to model. A semideterministic model, first developed for open, flat areas, has been generalized to accommodate any possible operational scenario and was tested in built-up areas. The model involves a stochastic part and a determinist which is a raytracing based part used to compute the long term mean (LTM) of the signal's coherent component.
\end{abstract}

\section{Keywords}

Low elevation links, nomadic user, statistical channel models, ray-tracing

\section{Introduction}

Unmanned Aerial Vehicles (UAV) will soon be widely used in civil applications as these systems offer a more economical and safer overview to the observer than helicopters or airplanes. By using 2 or $5 \mathrm{GHz}$ high frequency bands, more information can be transferred than at low frequencies which are primarily used for remotely commanding the UAV. However, such high frequency links undergo higher diffraction and shadowing/blockage losses [1], so new hardware is needed to transmit the information from a UAV to the command center ultimately leading developers to seek propagation channel models to define optimized architectures, modulations, coding, etc.

For low elevations between a UAV and the ground station, propagation characteristics lie between those of terrestrial and land mobile satellite links. Generative propagation channel models for land mobile satellite channels are reviewed in [2] while a survey of models for terrestrial propagation can be found in [3]. For UAV links, a universal propagation channel model is called for, one which is reliable and precise in all envisaged environments ranging from open to dense urban environments with deep shadowing conditions. A characterization of possible shadowing features, in terms of obstacle sizes and heights, is illustrated in Fig. 1 where buildings, trees, barns and walls are taken into account. Major terrain irregularity effects were not considered in this study as the chosen measurement scenarios were limited to flat areas.

A detailed path loss analysis is performed in [4], [5] and [6]. A modeling and experimental study for the open, flat areas was presented in [7] where a $2 \times 2$ MIMO semideterministic propagation channel model for UAVs with low elevation was first described. This semi-deterministic model is an evolution of a purely stochastic one developed for deep shadow conditions [8] applying a method from a vehicle-to-vehicle MIMO propagation channel model [9].

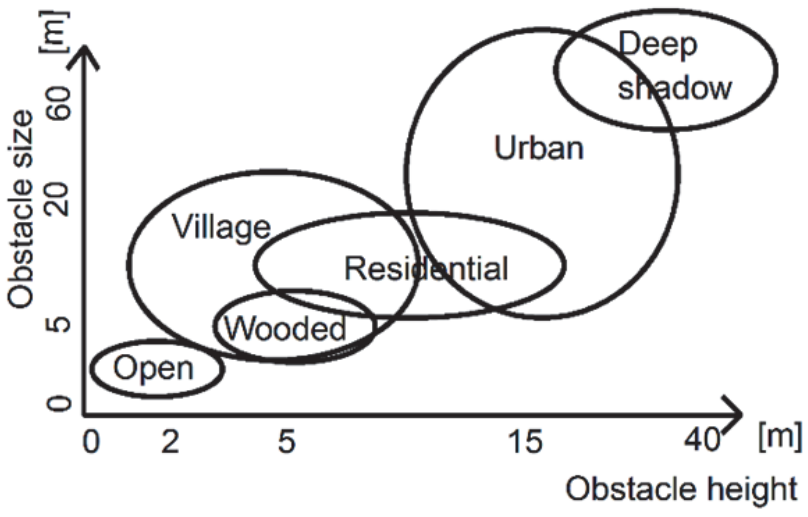

Fig. 1. Classification of studied environments as a function of obstacle size and height.

This paper aims to generalize the semi-deterministic model, originally developed for open areas [7], for more complex, cluttered scenarios such as built-up areas or areas such as those shown in Fig. 1. Contrary to what was observed for significantly deep shadow areas [8] where a purely stochastic approach was sufficient, there is a need for a deterministic, ray-tracing based component which cannot be reproduced by a purely statistical approach in open and cluttered areas. 


\section{Measurements}

Our measurement campaign was performed in the $2 \mathrm{GHz}$ band using a Multiple Input Multiple Output (MIMO) setup based on criteria laid out in [10] where channel separation was achieved through the use of two nearby carriers within the assumed channel's coherence bandwidth. A number of scenarios, as shown in Fig. 1, were measured [10]. However, in this paper attention was focused on a built-up area.

\subsection{Setup}

A remotely controlled airship, simulating a UAV and shown in Fig. 2, carried the transmitter (Tx) with two downward quarter-wave monopole antennas spaced $d=31.78 \mathrm{~cm}$ (over two wavelengths) apart.

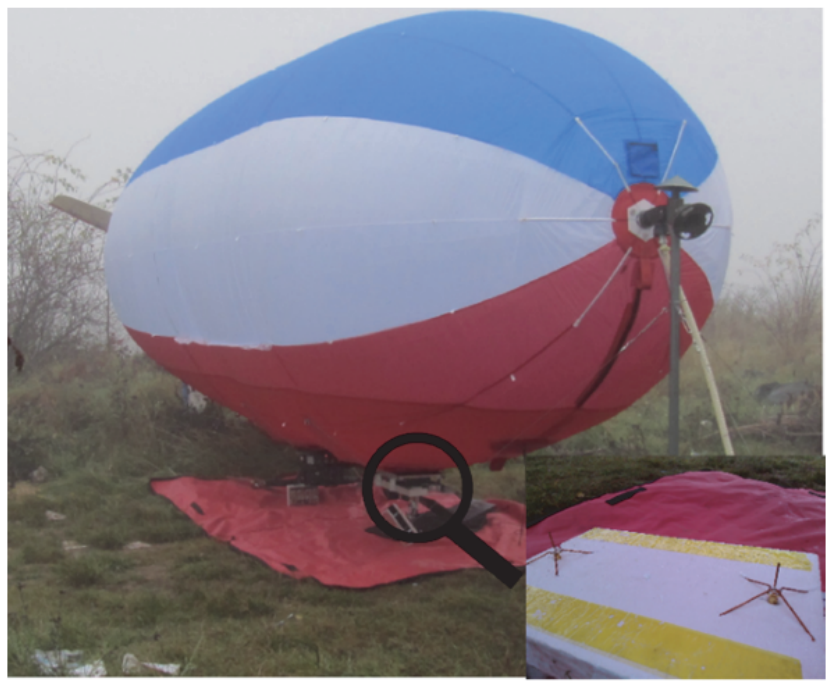

Fig. 2. Tx equipment, two monopole antennas, remote controlled airship.

Figure 3 shows the static receiver $(\mathrm{Rx})$ which is also composed of two monopole antennas with a four-rod ground plane. The monopoles were placed on a $1.5-\mathrm{m}$-high tripod with equal separation, $d=31.78 \mathrm{~cm}$, as on Tx. A $2 \times 2$ MIMO receiver acquired instantaneous power at a $10 \mathrm{kSps}$ (samples per second) rate. The measurement bandwidth of $12.5 \mathrm{kHz}$ accommodated the small Doppler shift, caused by airship motion, and provided a sufficiently small noise floor for a high dynamic range.

The $2 \times 2$ MIMO configuration is depicted in Fig. 4 . As in [11], the transmit frequencies were extremely close to one another $\left(F_{1}=2001.0666\right.$ and $\left.F_{2}=2000.8666 \mathrm{MHz}\right)$ and within the expected channel's coherence bandwidth, but far enough apart for the receiver's selectivity to separate the signals.

Only received power was measured as no phase information was recorded, i.e. $|h|^{2}$, where $h$ is the channel response at the carrier frequency. The measurements were narrowband since continuous wave $(\mathrm{CW})$ signals were used. This setup allowed the recording of sub-channels $\left|h_{11}\right|^{2},\left|h_{12}\right|^{2},\left|h_{21}\right|^{2}$ and $\left|h_{22}\right|^{2}$.

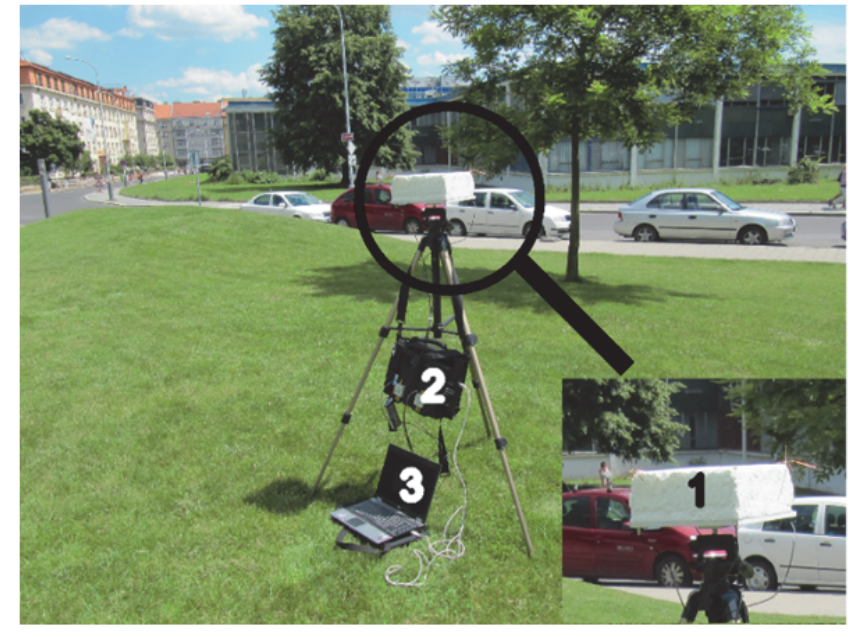

Fig. 3. Rx equipment: 1) Detail of antennas. 2) Receiver. 3) Computer with software.

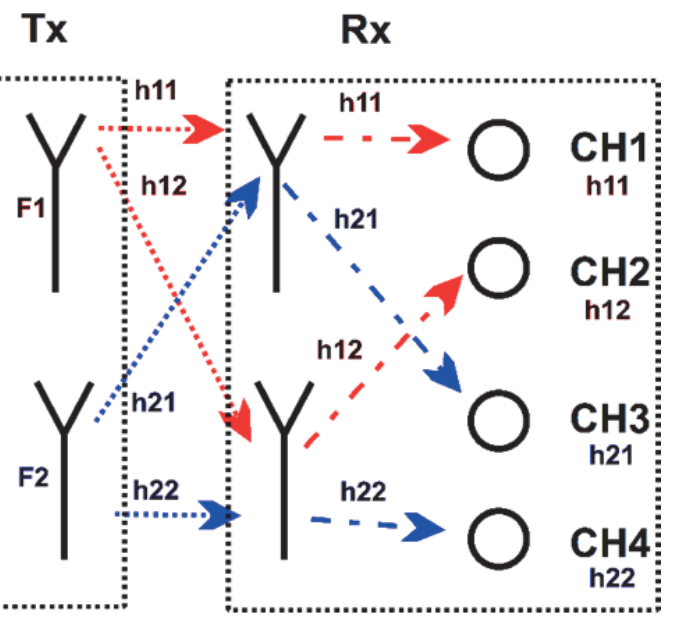

Fig. 4. $2 \times 2$ MIMO propagation channel schematic.

\subsection{Airship Trajectory}

Figure 5 depicts the airship trajectory with a maximum terrain height difference (without considering buildings) of $38 \mathrm{~m}$. The airship flew along the perimeter of a square with two parallel and two perpendicular flight paths with respect to Rx. The square sides were 200-mlong and the ground distance between $\mathrm{Rx}$ and $\mathrm{Tx}$ was approximately $2.7 \mathrm{~km}$. The airship speed was $5-8 \mathrm{~m} / \mathrm{s}$, so the small Doppler shift was covered by the receiver bandwidth,

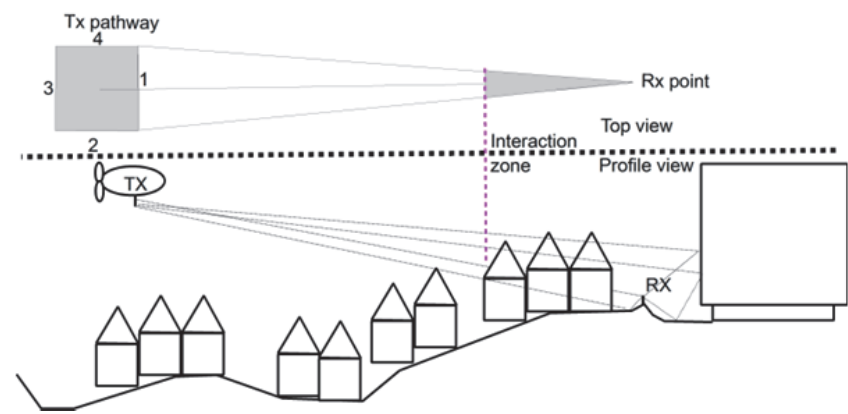

Fig. 5. Airship trajectory top view and projection onto side view. 
hence, it was neglected in further analysis. The elevation angle was set from $1^{\circ}$ to $3^{\circ}$, in $0.5^{\circ}$ increments, resulting in 20 flight paths. By performing ray-tracing, it was verified that all terrain interactions took place in the narrow shaded triangle close to $\mathrm{Rx}$, which means that, in practice, the relevant part of the terrain profile for all flight path transmit points was the same for all Tx positions as shown in Fig. 5.

\section{Versatile Propagation Channel Model}

Compared to the open field scenario [7] with two clear coherent contributions (direct and ground reflected rays), cluttered areas introduce more rays subject to diffraction and reflection which make up the coherent or geometry-dependent component of the proposed semi-deterministic model.

\subsection{Preliminary Requests}

In this paper we concentrated on the SISO case and, thus, analyzed one of the four available sub-channels. The others were used for consistency tests in the recorded signal.

Slight modifications to the model parameter extraction procedure used for open areas in [7] were introduced with the received power, $P(\mathrm{dBm})$, acquired at a sampling rate of $10 \mathrm{kSps}$. The signal was then decimated by a factor of 32 (window $1, W_{1}$ ). The sampling rate was, thus, reduced to $312.5 \mathrm{Sps}$ and yielded a new series, $P^{\prime}(\mathrm{dBm})$. The line-of-sight (LOS) power, $P_{\mathrm{LOS}}(\mathrm{dBm})$, was calculated using the link budget equation and subtracted from all four channels yielding the excess gain, $G(\mathrm{~dB})$, given by $G(\mathrm{~dB})=P^{\prime}-P_{\text {Los }}$. Note that $G(\mathrm{~dB})=10 \log 10|h|^{2}$.

The method of moments, MoM, [12] was applied to all four channel series. This technique assumes local Rice distributed variations and produces the following parameters: direct signal amplitude, $A$, and local average multipath power, $\Gamma$. A 16-sample window (window 2, $W_{2}$ ) was used to extract the coherent component amplitudes $A(\mathrm{~dB})$ and the incoherent component average powers $\Gamma(\mathrm{dB})$. The same window, $W_{2}$, was used for low-pass filtering of the $A$ and $\Gamma$ series by computing the running mean. A new series, $A^{\prime}(\mathrm{dB})$, resulted from subtracting the obtained long-term mean of $A, \operatorname{LTM}(A)$, i.e., $\mathrm{A}^{\prime}(\mathrm{dB})=\mathrm{A}-\operatorname{LTM}(A)$. Similarly, the series $\Gamma^{\prime}(\mathrm{dB})$ was obtained from $\Gamma(\mathrm{dB}), \Gamma^{\prime}(\mathrm{dB})=\Gamma-\operatorname{LTM}(\Gamma)+<\operatorname{LTM}(\Gamma)>$, where $<\operatorname{LTM}(\Gamma)>$ is the mean value of $\operatorname{LTM}(\Gamma)$.

A general model is used to model first- and secondorder characteristics of the propagation channel model for different scenarios. This model is composed of a deterministic part, whose structure changes with the scenarios, and a statistical part, whose structure remains the same with only the parameters being modified. Figure 6 shows the channel model's schematic diagram with greater detail of the model given in [7]. The channel model consists of

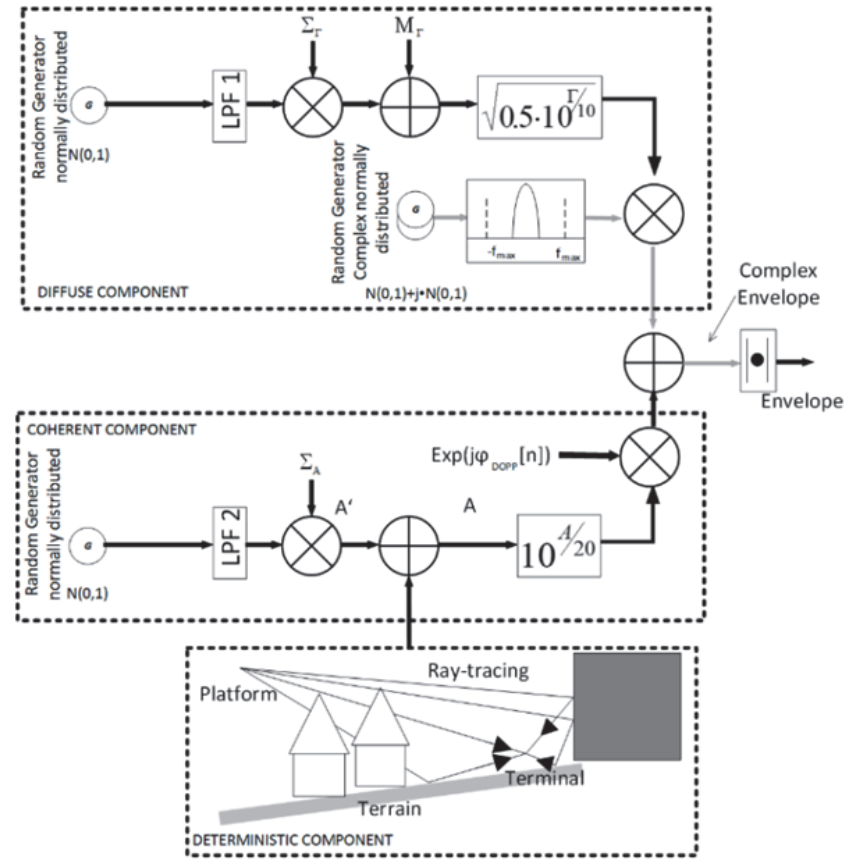

Fig. 6. SISO propagation channel model for an urban environment.

a coherent component divided into a geometry-dependent (deterministic) part and a slowly varying stochastic term, in addition to an incoherent component (diffuse multipath) representing the diffuse multipath contribution.

\subsection{Geometry-dependent Coherent Compo- nent and Simple Ray-tracing Calculation}

As there is a deterministic component in the received signal that cannot be reproduced by means of statistical techniques, it was necessary to perform simple ray-tracing to evaluate this coherent component, thus, limiting our analysis to a small number of Rx locations and elevation angles. Figure 7 shows an aerial photograph of Fleming Square, in Prague, with two yellow points indicating the measurement locations. The perimeter of the local environment (an open square in a built-up area) which is most likely to affect the propagation conditions is also shown.

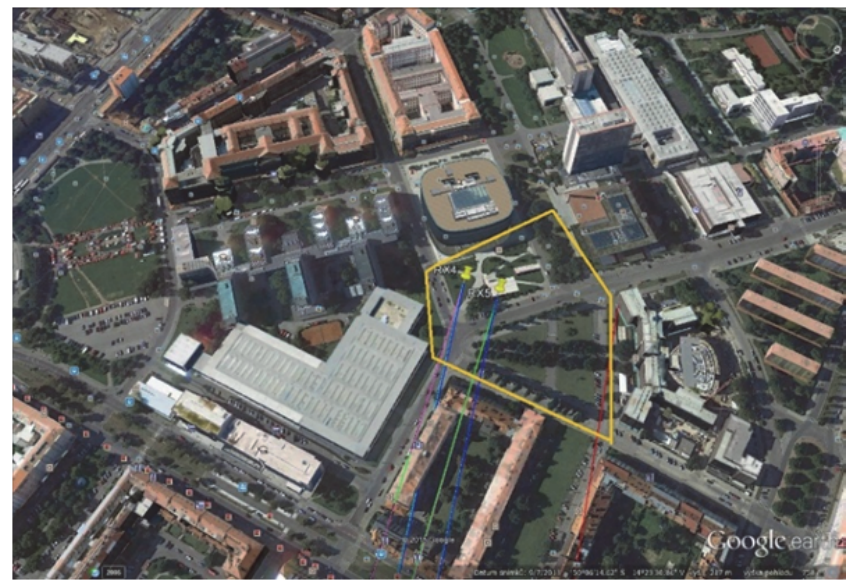

Fig. 7. Buildings close to Fleming Square (yellow outline) in Prague (Czech Republic). 
Blue and green lines show the paths from the corner points representing the flight path (Fig. 5) to the two Rx locations. The red line demonstrates how the crescent-shaped group of buildings on the right side of the photo of the perimeter cannot contribute side reflections significant enough to affect the received signal.

In this case, the $3 \mathrm{D}$ propagation conditions can be simplified to the vertical-plane 2D representation depicted in Fig. 8. As discussed in [8], the closest-to-Rx building's edge influences the received signal the most, hence, buildings beyond this point are neglected. The elements considered in the ray-tracing include a 15 -m-high building edge located $100 \mathrm{~m}$ from Rx which was placed on a 1.5-m-high tripod sitting on a 1-m-high hill. Behind Rx there is a modern building with a glass surface which is $21 \mathrm{~m}$ in height and $55 \mathrm{~m}$ away from $\mathrm{Rx}$. We calculated the power as the complex sum of four rays. The variations in each ray, as Tx moves, give rise to signal variations related to the computed value. Such variations were modeled statistically.

Figure 8 shows the magnitudes of the various rays, which were computed as follows: the first ray is a direct path undergoing diffraction; the second is a diffracted ground-reflected ray with a reflection coefficient of $\rho_{1}=-0.8$; the third is a diffracted-reflected ray with the reflection point located on the tinted-glass building behind Rx with a reflection coefficient of $\rho_{2}=-1$; the fourth reaches Rx via a reflection on the glass building $\left(\rho_{2}\right)$ and then the ground $\left(\rho_{1}\right)$. Due to an extremely low elevation angle, and its relative insignificance, the figure is not drawn in scale.

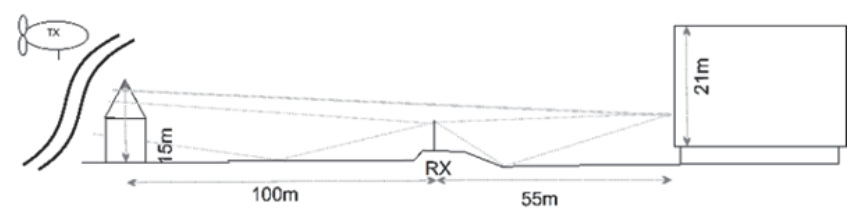

Fig. 8. Ray-tracing 2D diagram.

The diffraction loss can be calculated using a simple knife-edge model [13] where the diffraction parameter is given by

$$
v=h \sqrt{\frac{2}{\lambda} \cdot\left(\frac{1}{d_{1}}+\frac{1}{d_{2}}\right)}
$$

where $h$ is the difference between edge height and the intersection point of the LOS path with the knife-edge plane, $\lambda$ is wavelength, and $d_{1}$ and $d_{2}$ are the distances of Tx and $\mathrm{Rx}$ from the knife edge. The diffraction loss is $-F_{i}$ and is given by

$$
F_{i}=\frac{1+j}{2} \cdot\left(\left(\frac{1}{2}-C(v)\right)-j \cdot\left(\frac{1}{2}-S(v)\right)\right),
$$

where $C$ and $S$ are Fresnel integrals. The total received power, relative to LOS, is given by the complex sum of the four rays, namely

$$
g=\left|F_{1}+\rho_{1} F_{2}+\rho_{2} F_{3}+\rho_{1} \rho_{2} F_{4}\right|^{2},
$$

which is $L=20 \cdot \log 10(|g|)$, a diffraction loss in logarithmic units meaning this vector represents LTM (A), the coherent component series.

\subsection{Stochastic Element of the Coherent Component}

The stochastic component of the deterministic part discussed above, $\operatorname{LTM}(A)$, is modeled using a normal distribution in $\mathrm{dB}$ (log-normal in linear units). The autocorrelation function is approximated by a Gaussian function,

$$
R(\tau)=\exp \left(\frac{-\tau^{2}}{2 \tau_{A}^{2}}\right)
$$

where $\sqrt{2} \tau_{A}$ is the correlation time, $\sqrt{2} \tau_{A} v$ is the correlation length, and $v$ is the airship's velocity. LPF2 (Fig. 5) is an FIR filter with an impulse response such that

$$
h(t)=\sqrt{\frac{2}{\tau_{A}}} \exp \left(\frac{-t^{2}}{\tau_{A}^{2}}\right) .
$$

More details can be found in [7]. The zero mean filtered output is multiplied by the standard deviation $\Sigma_{A}(\mathrm{~dB})$, then added to the vector containing the geometry-dependent component and, finally, the result is converted to linear (voltage/field) units, i.e., $a=10^{A^{\prime} / 20}$.

\subsection{Diffuse Component}

The principle for diffuse component generation did not change. The multipath power, $\Gamma^{\prime}$, series generator is also based on filtering normally distributed random time series generated in a similar way as the coherent component. The impulse response of the FIR filter follows the same expression as (5), a detailed extraction of which is described in [4]. The output series is multiplied by the standard deviation, $\Sigma_{\Gamma}(\mathrm{dB})$, and the mean value, $\mathrm{M}_{\Gamma}(\mathrm{dB})$, is added. Series $\Gamma^{\prime}$, in logarithmic units, is converted to the linear units using $\sigma=\sqrt{0.510^{\Gamma^{\prime} / 10}}$ which then multiples the output of the two Gaussian random generators in quadrature to set the appropriate multipath power level. The two Gaussian generator outputs are first filtered to include the Doppler spreading effect. This low-pass Butterworth filter is obtained by a trial-and-error method, with the following specifications: $f_{\mathrm{PASS}}=1 \mathrm{~Hz} ; R_{\mathrm{PASS}}=0.5 \mathrm{~dB} ; f_{\mathrm{STOP}}=120 \mathrm{~Hz}$; and $R_{\mathrm{STOP}}=40 \mathrm{~dB}$ which, together, shape the signal spectrum in the desired way as in the measurements. The output is added to coherent part $a$ to produce the channel's complex envelope time series.

\subsection{Model Parameters}

Model parameters were obtained for two $\mathrm{Rx}$ locations at Fleming Square and are summarized in Tab. 1. They are 


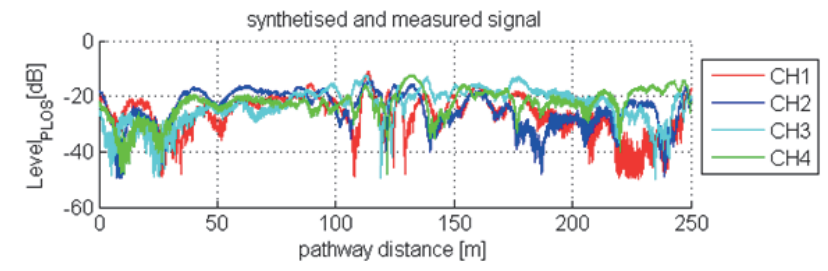

Fig. 9. Measured $2 \times 2$ MIMO time series.

\begin{tabular}{|c|c|c|c|c|c|c|c|c|}
\hline & \multicolumn{2}{|c|}{$1 \mathrm{deg}$} & \multicolumn{2}{|c|}{$2 \mathrm{deg}$} & \multicolumn{2}{|c|}{ 3 deg } & \multicolumn{2}{|c|}{ all } \\
\hline & Av. & Std. & Av. & Std. & Av. & Std. & Av. & Std. \\
\hline $\begin{array}{l}\sum_{\mathrm{A}^{\prime}} \\
(\mathrm{dB})\end{array}$ & 0.369 & 0.108 & 0.379 & 0.125 & 0.354 & 0.121 & 0.610 & 0.428 \\
\hline $\begin{array}{l}v \tau_{\mathrm{A}} \\
(\mathrm{m})\end{array}$ & 0.042 & 0.011 & 0.037 & 0.008 & 0.032 & 0.008 & 0.038 & 0.010 \\
\hline $\begin{array}{c}\mathrm{M}_{\Gamma} \\
(\mathrm{dB})\end{array}$ & $\begin{array}{c}- \\
46.26\end{array}$ & 1.96 & ${ }_{42.66}^{-}$ & 1.94 & $\begin{array}{c}- \\
41.77\end{array}$ & 1.88 & $\begin{array}{c}- \\
44.08\end{array}$ & 2.68 \\
\hline $\begin{array}{c}\sum_{\Gamma}^{\prime} \\
(\mathrm{dB})\end{array}$ & 1.391 & 0.230 & 1.353 & 0.262 & 1.288 & 0.222 & 1.352 & 0.242 \\
\hline $\begin{array}{l}v \tau_{\Gamma^{\prime}} \\
(\mathrm{m})\end{array}$ & 0.038 & 0.007 & 0.039 & 0.006 & 0.036 & 0.009 & 0.038 & 0.007 \\
\hline
\end{tabular}

Tab. 1. Model parameter ranges for different elevation angles.

notably similar as they are almost entirely independent of the elevation angle. The only parameter where the dependency on elevation angle is noticeable is the mean value of $\Gamma$ $\left(\mathrm{M}_{\Gamma}\right)$ which increases with elevation.

\section{Data Analysis and Discussion}

A long pathway with a mean elevation angle of $3.17^{\circ}$ is presented. Other series were fit similarly. The measured signal's $\operatorname{LTM}(A)$ vector was used in the synthesis to compare the results. The synthetized and measured signals are depicted in Fig. 10. In the deep nulls, the measured signal (blue curve) was clipped by the receiver's dynamic range, while the synthetized signal (red curve) was not clipped.

Figure 11 shows the histograms of the various model parameters extracted using the MoM technique [12]. As explained earlier, the signal is divided into a coherent part $A$ and a diffuse part represented by $\Gamma$. Parameters $A$ ' and $\Gamma$ ', derived from the previous ones, can be approximated by Gaussian distributions.

Vectors $\operatorname{LTM}(\Gamma)$ and $\operatorname{LTM}(A)$ were found to be uncorrelated. The behavior of $\operatorname{LTM}(A)$ can be described as the combination of four rays which can be identified and quantified using simple ray-tracing. $\operatorname{LTM}(\Gamma)$ was, as in [7], kept constant.

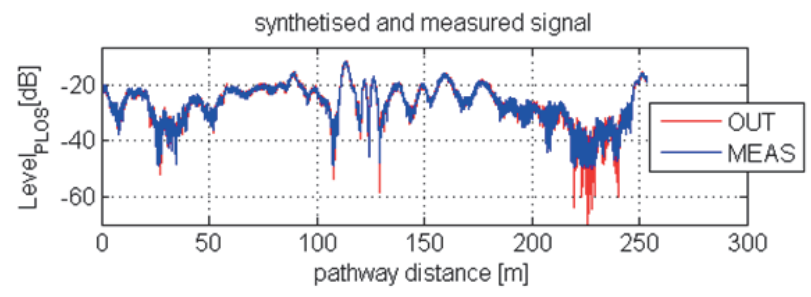

Fig. 10. Measured and synthetized signal for a long pathway with elevation angle $=3.17^{\circ}$.

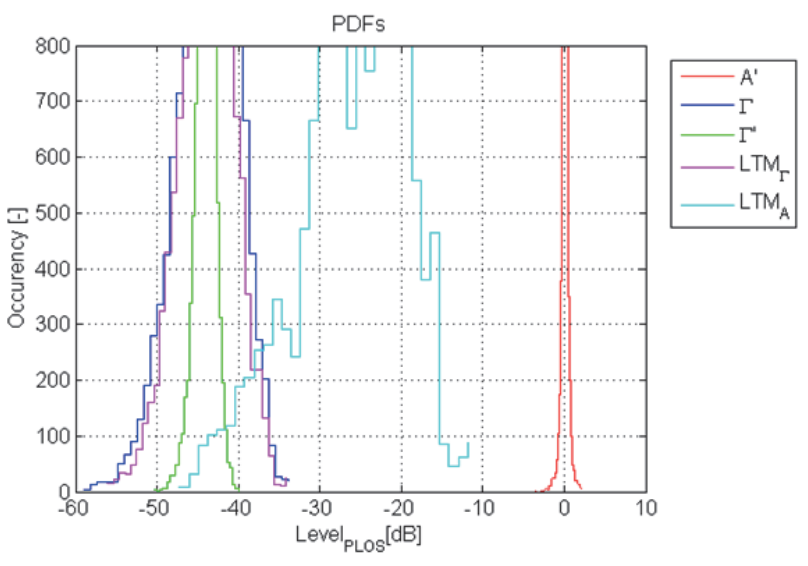

Fig. 11. Histogram of MoM parameter extraction results.
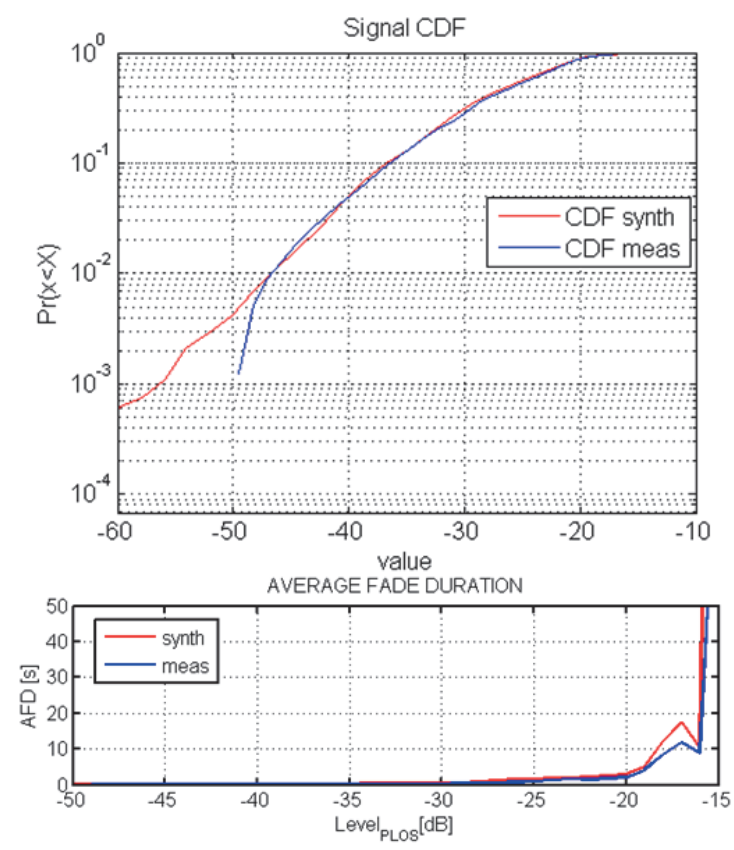

Fig. 12. Measured and synthetized signal a) CDF b) AFD.

Finally, Fig. 12 a) shows the cumulative distribution function (CDF) for the measured and synthetized signals. The differences at the tails of the distributions are due to the clipping of the deep nulls in the measurements. Fig. 12 b) shows the average fade duration (AFD) for measured and synthetized signal as having fair agreement.

\section{Conclusion}

In this paper a semi-deterministic channel model was developed for a built-up area and the obtained parameter set corresponding to the statistical part was computed. The geometry-dependent component was obtained using a simple $2 \mathrm{D}$ ray-tracing method. Four main rays were identified.

Values below $-121 \mathrm{dBm}(-121 \mathrm{dBm}$ absolute value $-44 \mathrm{~dB}$ in Level $_{\text {PLOS }}$ ) were clipped during the measurement. In the case of the synthetized signal, there was no need for clipping as shown in Fig. 10. 
The model is described with full parametrization for elevations angle from 1 to $3 \mathrm{deg}$. which supports the general purpose of this model. The model is valid for $1^{\text {st }}$-and $2^{\text {nd }}$-order characteristics such as CDF and AFD.

The model generality can be seen when the parameter ranges were only adapted in the stochastic part and the principle and schematic were not changed, even though the deterministic part was totally adapted to an urban scenario. This model enables us to synthetize the time series of one representation, so that when many representations are applied, similar statistics to the real measured channel are obtained.

\section{Acknowledgments}

This publication was supported by the European social fund within the framework of realizing the project "Support of inter-sectorial mobility and quality enhancement of research teams at Czech Technical University in Prague", CZ.1.07/2.3.00/30.0034.

\section{References}

[1] SAUNDERS, S., ARAGÓN-ZAVALA, A. Antennas and Propagation for Wireless Communication Systems. Wiley, 2007. ISBN: 978-0-470-84879-1

[2] PÉREZ-FONTÁN, F., MAYO, A., MAROTE, D., PRIETO-CARDEIRA, R., MARINO-ESPINEIRA, P., MACHADO, F., RIERA, $\mathrm{N}$. Review of generative models for the narrowband land mobile satellite propagation channel. International Journal on Satellite Communications, 2008, no. 26, p. 291-316. DOI: 10.1002/sat.914

[3] BARSOCCHI, P. Channel models for terrestrial wireless communications: a survey. CNR-ISTI Technical Report, April 2006.

[4] FENG, Q., MC GEEHAN, J, TAMEH, E. K., NIX, A. R. Path loss models for air-to-ground radio channels in urban environments. In Proc. of the 63rd IEEE Vehicular Technology Conference (VTC 06-Spring). Melbourne (Australia), May 2006, vol. 6, p. 2901 to 2905. DOI: 10.1109/VETECS.2006.1683399

[5] CERASOLI, C. An analysis of unmanned aerial vehicle relay coverage in urban environment. In Proc. of the IEEE Military Communications Conference (MILCOM 2007). Orlando (FL, USA), Oct. 2007, 7 p. DOI: 10.1109/MILCOM.2007.4455127

[6] FREW, E. W., BROWN, T. X. Airborne communication networks for small unmanned aircraft systems. Proceedings of the IEEE, Dec. 2008, vol. 96, no. 12, p. 2008-2027. DOI: 10.1109/JPROC.2008.2006127

[7] ZELENÝ, J., PÉREZ-FONTÁN, F., PECHAC, P., MARINOESPINEIRA, P. Open area $2 \times 2$ MIMO channel model for $2 \mathrm{GHz}$ low elevation links with diversity and capacity applications. IEEE Transactions on Antennas and Propagation (submitted).

[8] SIMUNEK, M, PÉREZ-FONTÁN, F., PECHAC, P. The UAV low elevation propagation channel in urban areas: Statistical analysis and time-series generator. IEEE Transactions on Antennas and Propagation, July 2013, vol. 61, no. 7, p. 3850-3858 DOI: 10.1109/TAP.2013.2256098
[9] KAREDAL, J., TUFVESSON, F., CZINK, N., PAIER, A., DUMARD, C., ZEMEN, T., MECKLENBRÄUKER, C. F., MOLISH, A. F. A geometry-based stochastic MIMO model for vehicle-to-vehicle communications. IEEE Transactions on Wireless Communications, July 2009, vol. 8, no. 7, p. 3646-3657. DOI: 10.1109/TWC.2009.080753

[10] ZELENÝ, J., PÉREZ-FONTÁN, F., PECHAC, P. Initial results from a measurement campaign for low elevation angle links in different environments. In Proceedings of the 9-th European Conference on Antennas and Propagation (EuCAP). Lisbon (Portugal), April 2015, 4 p.

[11] LACOSTE, F., CARVALHO, F., PÉREZ-FONTÁN, F., NUNEZFERNANDEZ, A., FABBRO, V., SCOT, G. MISO and SIMO measurements of the Land Mobile Satellite propagation channel at S-band. In Proceedings of the 4th European Conference on Antennas and Propagation (EuCAP). Barcelona (Spain), 2010, 5 p.

[12] GREENSTEIN, L. J., GHASSEMZADEH, S. S., ERCEG, V., MICHELSON, D. G. Ricean K-factors in narrowband fixed wireless channels: Theory, experiments, and statistical models. IEEE Transactions on Vehicular Technology, Oct. 2009, vol. 58, p. 4000-4012. DOI: 10.1109/TVT.2009.2018549

[13] ITU recommendation ITU-R P.526-11. [Online] Available at: www.itu.org

\section{About the Authors ...}

Jan ZELENÝ (corresponding author) was born in Prague. $\mathrm{He}$ received his Master degree from ENST Bretagne in 2007 and CTU Prague in 2008 as well as a Ph.D. as a collaboration between the University of LILLE I and MINATEC. His research interests include system engineering for radio architectures, integrated circuit design and propagation channel models.

Fernando PÉREZ-FONTÁN was born in Villagarcía de Arousa, Spain. He received a degree in Telecommunications Engineering and a Ph.D. degree from the Technical University of Madrid, Madrid, Spain, in 1982 and 1992, respectively. After working in the industry since 1984, he became an Assistant Professor at the University of Vigo, Galicia, Spain, in 1988. In 1993, he became an Associate Professor and, in 1999, a full Professor with the Signal Theory and Communications Department of the University of Vigo. He is the author of a number of books and journal papers and has been the leader in several projects funded by public and private entities. He participates in ITU-R WG3 on Propagation Modeling. He has been a Management Committee Member of EU COST 255, 280, 297, and IC0802.

Pavel PECHAC (M'94-SM'03) was born in Prague, Czech Republic. He received his M.Sc. degree and Ph.D. degree in Radio Electronics from the Czech Technical University, Prague, Czech Republic, in 1993 and 1999, respectively. He is currently a Professor in the Department of Electromagnetic Field at the Czech Technical University, Faculty of Electrical Engineering. His research interests are in the field of radio wave propagation and wireless systems. More details can be found at http://pechac.elmag.org. 